\title{
Interplay of Crystal Structure Preference and Magnetic Ordering in High Entropy CrCoFeNiAl Alloys
}

\author{
K. JASIEWICZ*, S. KAPRZYK, J. TOBOLA \\ AGH University of Science and Technology, Faculty of Physics and Applied Computer Science, \\ al. Mickiewicza 30, 30-059 Krakow, Poland
}

\begin{abstract}
In this work, some relations between crystal structure and magnetic ordering in CrCoFeNiAl HEAs are discussed in view of the KKR-CPA calculations. Remarkably, it is noticed that the transition between $f c c$ and $b c c$ phases in the analysed $\mathrm{CrCoFeNi}_{x} \mathrm{Al}$ and $\mathrm{CrCoFeNiAl}_{x}$ alloys is closely related to change in ordering of the local magnetic moments, namely the magnetic moment on $\mathrm{Cr}$ is either parallel (ferromagnetic) or antiparallel (ferrimagnetic) to the magnetic moments of other atoms ( $\mathrm{Co}, \mathrm{Fe}$ and $\mathrm{Ni}$ ). On the whole, the theoretical diagram showing the $f c c-b c c$ phase preference and their coexistence vs. alloy composition well corroborates with available experimental data.
\end{abstract}

DOI: 10.12693/APhysPolA.133.511

PACS/topics: 71.15.Nc, 71.20.Be, 71.23.-k, 75.50.Bb

\section{Introduction}

In the recent years high entropy alloys (HEA) [1, 2] became a subject of intense experimental and theoretical investigations, since in contrast to traditional complex alloys usually consisting of one, two or three main elements, HEAs may contain over a dozen of constitutent elements with comparable atomic contributions. Surprisingly, simple structures as bcc, fcc or hcp, including randomly distributed atoms on crystallographic sites, were found to be structural models well describing the atoms ordering in HEAs. The configurational entropy of such $N$ - element system, usually expressed as $S_{\text {conf }}=-k_{\mathrm{B}} \Sigma_{i=1}^{N} c_{i} \ln c_{i}$ (where $c_{i}$ represents concentration of $i$-th element and $k_{\mathrm{B}}$ is Boltzmann constant) is relatively high and reaches the maximum value when the concentrations of each element are the same, i.e. $c_{i}=1 / N$ yielding $S_{\text {conf }}=k_{\mathrm{B}} \ln N$.

TABLE I

Relations between element concentration $c_{M}, x$ value and $c_{\mathrm{Al}} / \mathrm{c}_{\mathrm{Ni}}$ ratio. $M$ stands for $\mathrm{Al}$ in $\mathrm{Al}_{x}$ and $\mathrm{Ni}$ in $\mathrm{Ni}_{x}$ alloy.

\begin{tabular}{c|c|c|c}
\hline \hline$c_{M}[\mathrm{at} \%]$ & $x$ & $c_{\mathrm{Al}} / c_{\mathrm{Ni}}\left(\mathrm{Al}_{x}\right)$ & $\mathrm{c}_{\mathrm{Al}} / c_{\mathrm{Ni}}\left(\mathrm{Ni}_{x}\right)$ \\
\hline 10 & 0.44 & 0.44 & 2.27 \\
15 & 0.71 & 0.71 & 1.41 \\
20 & 1.00 & 1.00 & 1.00 \\
25 & 1.33 & 1.33 & 0.75 \\
30 & 1.71 & 1.71 & 0.58 \\
35 & 2.15 & 2.15 & 0.46
\end{tabular}

At very beginning, experimental investigations concerned the crystal structures, phase formation and pref-

\footnotetext{
* corresponding author; e-mail kinga.jasiewicz@fis.agh.edu.pl
}

erence as well as conditions of their relative stability. The fact that such complex, disordered systems crystallize in very simple lattices prompted many research groups to study more fundamental aspects of HEAs as electronic, transport or magnetic properties. Noteworthy, many magnetic HEAs are based on quaternary CrCoFeNi system, which is often considered as the simplest HEA. Accordingly, experimental and theoretical results of studies of $\mathrm{CrCoFeNiAl}_{x}(0<x<2.5)$ [3-6], CrCoFeNiGe [7], CrCoFeNiGa [8], CoCrFeMnNi [9] or $\mathrm{CoCrFeNiPd}_{x}$ [10] have been recently reported.
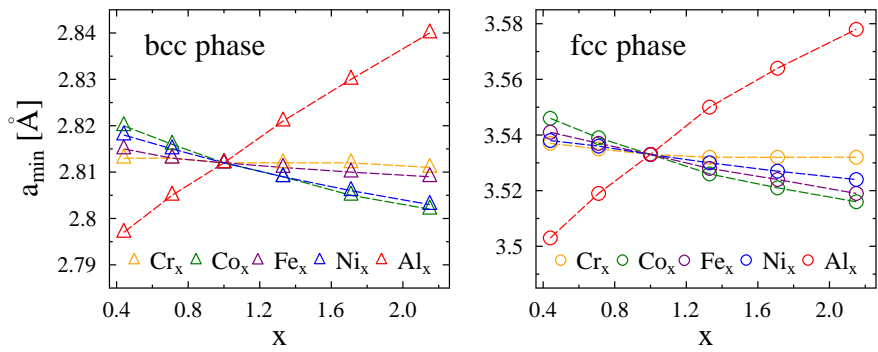

Fig. 1. Calculated lattice constants of $\mathrm{CrCoFeNiAl}$ systems (in $b c c$ and $f c c$ phases) vs. $x$ value.

This work extends the previous theoretical studies $[10,11]$, focusing mostly on structural, electronic and magnetic properties in the CrCoFeNiAl HEAs. Based on results of the Korringa-Kohn-Rostoker (KKR) calculations combined with the coherent potential approximation (CPA) to account for the multi-element chemical disorder, we report some correlations between magnetic moments and their mutual ordering on transition element atoms on one hand, and the $b c c / f c c$ phase preference (or coexistence) on the other one. The KKR-CPA has been already successfully applied to study the phase preference in $\mathrm{CrCoFeNiAl}_{x}$ system [11] as well as to interpret the superconductivity in TaNbHfZrTi HEA [12, 13]. 

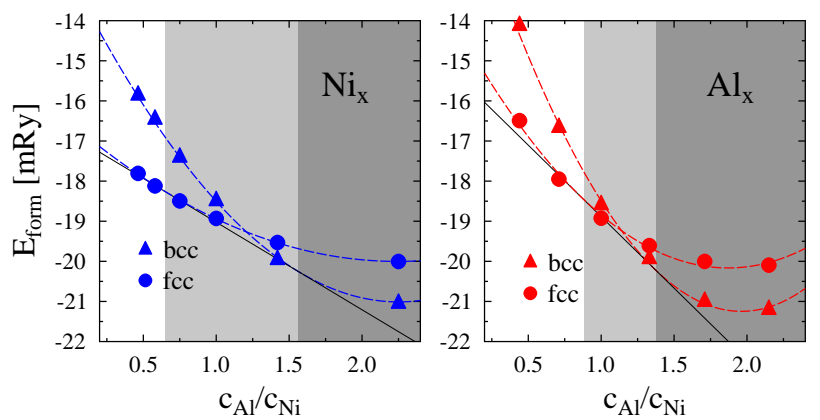

Fig. 2. Formation energies of $\mathrm{CrCoFeNiAl}_{x}$ and $\mathrm{CrCoFeNi}_{x} \mathrm{Al}$ alloys from the KKR-CPA method.

Here, the crystal potential of muffin-tin form was constructed in the framework of the local spin density approximation (LSDA) and the charge- and spinselfconsistent KKR-CPA [14] iterations were repeated until reaching the convergence of potentials and charges below $0.1 \mathrm{mRy}$ and $10^{-3} e(e-$ electron charge), respectively. Total, site-decomposed and $l$-decomposed (with angular momentum cut-off $l_{\max }=3$ ) density of states, were calculated using tetrahedron $\boldsymbol{k}$-space integration technique, generating $\sim 1200$ tetrahedrons and $\sim 300 \boldsymbol{k}$ point grid in the irreducible part of Brillouin zone. The Fermi level was precisely determined from the Lloyd formula [15].

\section{Results and discussion}

The KKR-CPA calculations were performed for five systems: $\mathrm{Cr}_{x} \mathrm{CoFeNiAl}\left(\mathrm{Cr}_{x}\right), \quad \mathrm{CrCo}_{x} \mathrm{FeNiAl}$ $\left(\mathrm{Co}_{x}\right), \mathrm{CrCoFe}_{x} \mathrm{NiAl}\left(\mathrm{Fe}_{x}\right), \mathrm{CrCoFeNi}_{x} \mathrm{Al}\left(\mathrm{Ni}_{x}\right)$ and $\mathrm{CrCoFeNiAl}_{x}\left(\mathrm{Al}_{x}\right)$, where the $x$ value was changed from 0.44 to 2.15 , which is equivalent to variation of the element concentration from 10 to $35 \%$ (see Table I). The equilibrium lattice constants obtained from the total energy calculations are gathered in Fig. 1. The difference between computed and available experimental values [16] does not exceed typical LDA error.

The formation energy of all considered HEA compositions (in both phases) was calculated from the wellknown formula $E_{\text {form }}^{f c c / b c c}=E_{H E A}^{t o t}-\Sigma_{i}^{N} c_{i} E_{i}$, where $E_{H E A}^{t o t}$ is total energy obtained at equilibrium lattice constant in $b c c$ or $f c c$ phases, while $c_{i}$ and $E_{i}$ represent concentration and total energy of $i$-th element bulk solid, respectively.

In the whole range of concentrations, all calculated formation energies turned out to be negative, what may tentatively support the crystal stability of the $\mathrm{CrCoFeNiAl}$ HEAs. Since the difference between $E_{\text {form }}^{b c c}$ and $E_{\text {form }}^{f c c}$ computed for $\mathrm{Cr}_{x}, \mathrm{Co}_{x}$ and $\mathrm{Fe}_{x}$ systems was found to be as small as few $10^{-5} \mathrm{Ry}$, we decided to focus on $\mathrm{Al}_{x}$ and $\mathrm{Ni}_{x}$ alloys (Fig. 2), where the variation of formation energy is more pronounced. To allow for comparison of these two series, the $x$ variable was replaced with more convenient $c_{\mathrm{Al}} / c_{\mathrm{Ni}}$ ratio. In spite of quite different electronic configurations of $\mathrm{Al}$ and $\mathrm{Ni}$ atoms, the correspond- ing differences between $E_{\text {form }}^{b c c}$ and $E_{\text {form }}^{f c c}$ in both alloys are very close, suggesting that $c_{\mathrm{Al}} / c_{\mathrm{Ni}}$ ratio may have a strong impact on the $b c c / f c c$ phase preference. Using the KKR-CPA $x$-dependent energy formation obtained in $b c c$ and $f c c$ phases and employing common tangent line rule, the range of phase coexistence was estimated. The regions of single $f c c$ phase appearance (white area) or bcc (grey area) are separated by the region of their coexistence (light-grey area).

In spite of overall similarities of both HEA series, one notices that the $f c c-b c c$ phase transition appears for $\mathrm{c}_{\mathrm{Al}} / \mathrm{c}_{\mathrm{Ni}}=0.65$ ratio $\left(c_{\mathrm{Ni}}=14 \%\right)$ in $\mathrm{Ni}_{x}$ alloy, which is slightly smaller than the corresponding value 0.88 $\left(c_{\mathrm{Al}}=18 \%\right)$ in $\mathrm{Al}_{x}$ one. Also, in $\mathrm{Ni}_{x}$ the range of the $f c c$ - bcc phase coexistence is broader than in $\mathrm{Al}_{x}$. Furthermore, the phase preference changes in favor of the $b c c$ structure for the $c_{\mathrm{Al}} / c_{\mathrm{Ni}}$ ratios $1.56\left(c_{\mathrm{Ni}}=28 \%\right)$ and $1.38\left(c_{\mathrm{Al}}=26 \%\right)$ in $\mathrm{Ni}_{x}$ and $\mathrm{Al}_{x}$, respectively. To our best knowledge, experimentally the range of the $f c c-b c c$ phase coexistence was investigated only for $\mathrm{Al}_{x} \mathrm{HEA}$ and both phases were detected for $\mathrm{Al}$ concentration between $11 \%$ and $23.8 \%$ [16], while in other work [17] the coexistence range was found to be dependent on sample preparation method, namely either $10 \div 18 \%$ or $7 \div 23 \%$, for as cast and homogenized alloys, respectively. Noteworthy, KKR-CPA results also remain in good agreement with previous theoretical calculations $(14 \div 24 \%)$ [18].

As far as the magnetic properties are concerned, KKRCPA calculations show that total magnetic moment in $b c c$ phase $\mu_{W S}^{b c c}$ is larger than the $\mu_{W S}^{f c c}$ value and the highest contributions arise from $\mathrm{Fe}$ and $\mathrm{Co}$ atoms, both in the $\mathrm{Al}_{x}$ and $\mathrm{Ni}_{x}$ series (Fig. 3). Besides, one can notice that except of $\mu_{\mathrm{Ni}}^{b c c}$ in $\mathrm{Ni}_{x}$ alloy (which tends to slightly increase with $x$ ) all local magnetic moments decrease (in absolute value) with increasing $c_{\mathrm{Al}} / c_{\mathrm{Ni}}$ ratio. However, some differences between two series can be notified, since in the $b c c$ phase local magnetic moments in the $\mathrm{Al}_{x}$ series decrease more rapidly than the corresponding values in the $\mathrm{Ni}_{x}$ series. In case of the $f c c$ phase, tendency is opposite and one observes faster change of $\mu_{\mathrm{M}}^{f c c}(\mathrm{M}=\mathrm{Cr}$, $\mathrm{Co}, \mathrm{Fe}, \mathrm{Ni}$ ) in $\mathrm{Ni}_{x}$ systems with respect to the $\mathrm{Al}_{x}$ ones. In both systems the computed magnetic moments of $\mathrm{Fe}$, Co and Ni atoms are coupled parallel (bcc and $f c c$ ) in the whole range of $c_{\mathrm{Al}} / c_{\mathrm{Ni}}$ ratio, while the variation of $\mathrm{Cr}$ magnetic moment with HEA composition is more complex: for large $c_{\mathrm{Al}} / c_{\mathrm{Ni}}$ ratio the moment was found to be oriented antiparallel to moments on other $3 d$ atoms, whereas it changes the polarisation when the $c_{\mathrm{Al}} / c_{\mathrm{Ni}}$ ratio is decreasing. Looking closer at shadowed backgrounds (Fig. 3) one may conclude that in $\mathrm{Al}_{x}$ and $\mathrm{Ni}_{x}$ HEA series the critical $c_{\mathrm{Al}} / c_{\mathrm{Ni}}$ ratio for which the $\mu_{C r}^{b c c}$ changes sign, almost coincides with the border composition of the single $f c c$ phase occurence. In view of the KKR-CPA predictions of the phase preference, it seems that the Cr magnetic moment shows a strong propensity to be aligned antiparallel to moments on other transition metal atoms, both in $b c c$ and $f c c$ phases. 

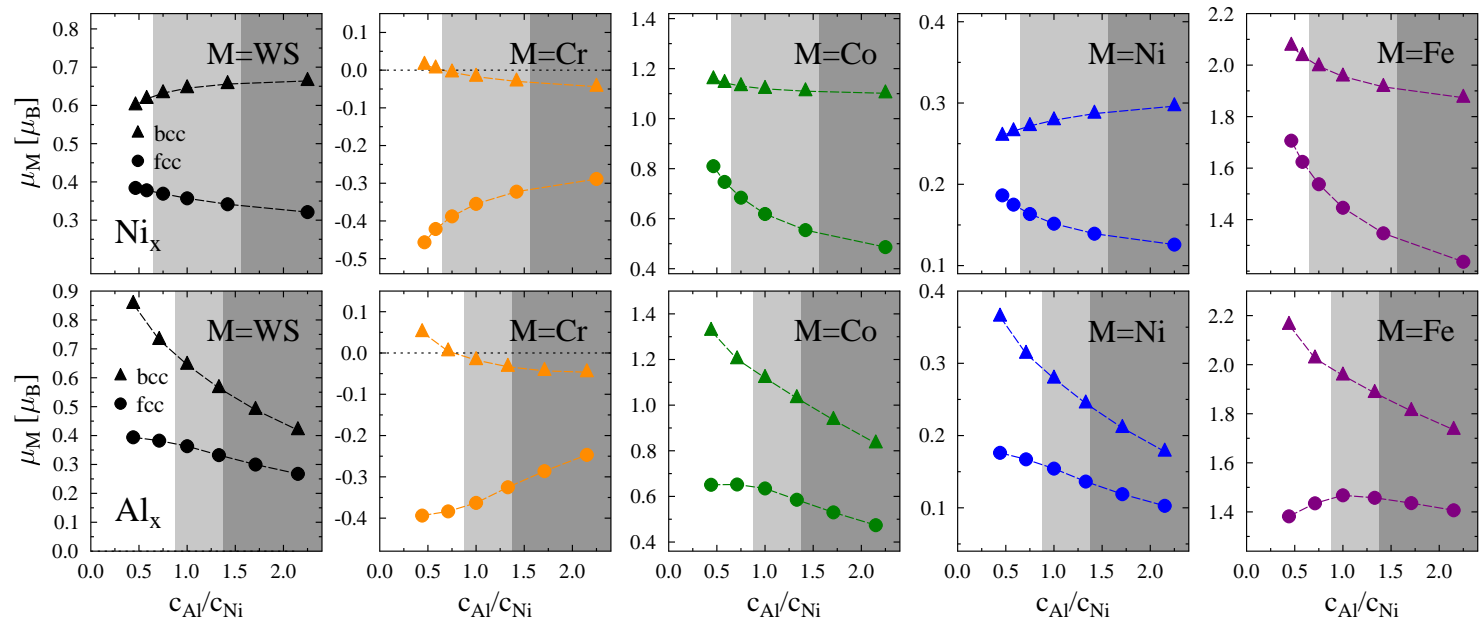

Fig. 2. Variation of average magnetic moment per Wigner-Seitz cell and local magnetic moments on consistuent atoms in $\mathrm{CrCoFeNiAl}_{x}$ and $\mathrm{CrCoFeNi}_{x} \mathrm{Al}$ alloys from KKR-CPA calculations.

\section{Conclusions}

The results of the spin-polarised KKR-CPA calculations of the CrCoFeNiAl HEAs are presented for compositions in wide range of all element concentrations. The $f c c-b c c$ phase preference as well as the phase coexistence were discussed in view of the formation energy analysis. A special attention was paid to $\mathrm{Al}_{x}$ and $\mathrm{Ni}_{x}$ series, where formation energy was found to be particularly sensitive to $x$. The theoretical phase diagram as well as critical composition of phase transitions quite well corroborate with available experimental data. The variation of magnetic properties with composition $\left(c_{\mathrm{Al}} / c_{\mathrm{Ni}}\right.$ ratio) in the $\mathrm{CrCoFeNi}_{x} \mathrm{Al}$ and $\mathrm{CrCoFeNiAl}_{x}$ HEA series is rather complex, since total magnetic moment slightly decreases in both cases in $f c c$ phase, while it changes in opposite way (increases in $\mathrm{Ni}_{x}$ and decreases in $\mathrm{Al}_{x}$ ) in $b c c$ phase. Noteworthy, in view of the KKR-CPA results, the composition limit of the single $f c c$ phase occurence, both in $\mathrm{CrCoFeNi}_{x} \mathrm{Al}$ and $\mathrm{CrCoFeNiAl}_{x}$ series is found to be quite close to the $c_{\mathrm{Al}} / c_{\mathrm{Ni}}$ ratio when $\mathrm{Cr}$ magnetic moment changes its orientation with respect to the moments on $\mathrm{Fe}$, $\mathrm{Co}$ and $\mathrm{Ni}$ atoms. However, $\mathrm{Cr}$ moment tends to be aligned antiparallel to other $3 d$ moments in the energetically prefered phases.

\section{Acknowledgments}

This work was partly supported by the NCN project (No. UMO-2015/17/B/ST3/01204) and the Ministry of Science and Higher Education in Poland.

\section{References}

[1] J.W. Yeh, S.K. Chen, S.J. Lin, J.Y. Gan, T.S. Chin, T.T. Shun, C.H. Tsau, S.Y. Chang, Adv. Eng. Mater 6, 299 (2004).

[2] J.W. Yeh, Y.L. Chen, S.J. Lin, S.K. Chen, Mater. Sci. Forum 560, (2007).

[3] Y.F. Kao, S.K. Chen, T.J. Chen, P.C. Chu, J.W. Yeh, S.J. Lin, J. Alloys Compd. 509, 1607 (2011).
[4] M.S. Lucas, L. Mauger, J.A. Muňoz, Y. Xiao, A.O. Sheets, S.L. Semiatin, J. Horwath, Z. Turgut, J. Appl. Phys. 109, (2011).

[5] M.S. Lucas, D. Belyea, C. Bauer, N. Bryant, E. Michel, Z. Turgut, S.O. Leontsev, J. Horwath, S.L. Semiatin, M.E. McHenry, C.W. Miller, J. Appl. Phys. 113 (2013).

[6] C. Niu, A.J. Zaddach, A.A. Oni, X. Sang, J.W. Hurt III, J.M. LeBeau, C.C. Koch, D.L. Irving, Appl. Phys. Lett. 106, 161906 (2015).

[7] S. Huang, A. Vida, D. Molnár, K. Kádas, L.K. Varga, E. Holmström, L. Vitos, Appl. Phys. Lett. $\mathbf{1 0 7}$, 251906 (2015).

[8] S. Huang, W. Li, X. Li, S. Schönecker, L. Bergqvist, E. Holmström, L.K. Varga, L. Vitos, Materials $\& \mathcal{E} D e-$ sign 103, 71 (2016).

[9] D. Ma, B. Grabowski, F. Körmann, J. Neugebauer, D. Raabe, Acta Mater. 100, 90 (2015).

[10] M. Calvo-Dahlborg J. Cornide, J. Tobola, D. NguyenManh, J. S. Wróbel, J. Juraszek, S. Jouen, U. Dahlborg, J. Phys. D: Appl. Phys. 50, 18 (2017).

[11] K. Jasiewicz, J. Cieślak, S. Kaprzyk, J. Tobola, J. Alloys Compd. 648, 307 (2015).

[12] P. Koželj, S. Vrtnik, A. Jelen, S. Jazbec, Z. Jagličić S. Maiti, M. Feuerbacher, W. Steurer, J. Dolinšek, Phys. Rev. Lett. 113, 107001 (2014).

[13] K. Jasiewicz, B. Wiendlocha, P. Korbeń, S. Kaprzyk, J. Tobola, Phys. Status Solidi RRL 10, 415 (2016).

[14] A. Bansil, S. Kaprzyk, P.E. Mijnarends, J. Tobola, Phys. Rev. B 60, 13396 (1999).

[15] S. Kaprzyk, A. Bansil, Phys. Rev. B 42, 7358 (1990).

[16] H.P. Chou, Y.S. Chang, S.K. Chen, J.W. Yeh, Materials Science and Engineering B 163, 184 (2009).

[17] Y.F. Kao, S.K. Chen, T.J. Chen, J.W. Yeh, S.J. Lin, J. Alloys Compd. 488, 57 (2009).

[18] F. Tian, L. Delczeg, N. Chen, L.K. Varga, J. Shen, L. Vitos, Phys. Rev. B 88, 085128 (2013). 